\title{
Performance Analysis of OFDM Systems with Gaussian Distributed Phase and Frequency Offsets
}

\author{
Sabitha Gauni, J. Elakkiya, R. Kumar, Vidhyacharan Bhaskar \\ Department of Electronics and Communication Engineering \\ SRM University, Kattankulathur-603203, \\ Tamilnadu, India.
}

\begin{abstract}
In this paper, a theoretical analysis to evaluate the performance of Orthogonal Frequency Division Multiplexing (OFDM) systems under the combined influence of Phase Offset and Frequency Offset over Rayleigh, Weibull and Nakagami fading channels using Binary Phase Shift Keying (BPSK) Modulation is presented. The performance of an OFDM system is degraded by both the Frequency Offset and the Phase Offset. The degradation of Bit Error Rate (BER) caused by the presence of Phase Offset and Frequency Offset is analytically evaluated. Hence, closed form expressions are derived to compute the BER with Rayleigh, Weibull and Nakagami fading channels assuming Gaussian probability density function of the Frequency Offset and Phase Offset. BER performance results are evaluated numerically following the analytical approach.
\end{abstract}

\section{Keywords}

Orthogonal Frequency Division Multiplexing (OFDM); Phase Offset; Frequency Offset; Bit Error Rate (BER); Probability Density Function (PDF); Signal to Noise Ratio (SNR).

\section{INTRODUCTION}

OFDM has become a popular technique for transmission in wireless communication systems due to its high data rate transmission capability with high bandwidth efficiency and robustness to multipath fading and delay. It has also been proposed as the core technique for fourthgeneration (4G) mobile communications. As OFDM makes use of the spectrum efficiently, it has been widely adopted and implemented in wired and wireless communications, such as Digital Subscriber Line (DSL), European Digital Audio Broadcasting (DAB), Digital Video Broadcasting-Terrestrial (DVB-T), its handheld version DVB-H, and IEEE 802.11a/g standards for Wireless Local Area Networks (WLANs) etc [1],[2]. OFDM systems have become significant because they are robust to frequency selective fading. As OFDM systems have low equalization complexity the cost of FFT techniques implementation is reduced. But, the OFDM systems are more sensitive to frequency synchronization errors and Phase noise than single carrier systems [3]. The presence of Frequency Offset and Phase noise disturbs the orthogonality between the carriers, thereby causing Inter Carrier Interference (ICI). Hence, there is a need to analyze the performance of such systems [3].
Frequency offset is a deterministic phenomenon, which is usually caused by different carrier frequencies of the transmitter and receiver, or by Doppler shift. On the other hand, phase noise is a random process caused by frequency fluctuations at the receiver and transmitter oscillators. The principles of analyzing the frequency offset effects have been described in [5], [6].

Several works discussing the error probability of OFDM systems with Carrier Frequency Offset (CFO) can be found in literature [3]-[5]. In this context, Moose [6] gives the maximum likelihood estimator of the CFO, based on the observation of two consecutive and identical symbols. The maximum offset that can be handled is $\pm 1 / 2$ the subcarrier spacing. However, analytical expressions were given for the first time in [7] for Binary Phase Shift Keying (BPSK) OFDM systems with CFO in AWGN, flat and frequency selective Rayleigh fading channels.

Although the BER expression derived therein is acceptable for all values of CFO for AWGN channels, the same is not true for flat and frequency selective channels. This is because the expressions have been derived assuming the argument of $\mathrm{Q}$ function in the expressions (7) and (32) of [7] to be positive. This is not true for higher values of CFO, leading to a mismatch between the theoretical and actual Bit Error Rate (BER) in such cases. Performance of an OFDM system in presence of CFO and phase noise has been analyzed in several research works during the last few years. The BER performance evaluation of an OFDM system in the presence of CFO and phase noise is shown in [8].

The main objective of this paper is to derive accurate BER expressions valid for all values of Phase and Frequency Offsets for BPSK OFDM systems. We first calculate the PDF of various fading distributions in an integral form and then use this PDF to evaluate the error rate performance for BPSK modulated OFDM system in Rayleigh, Weibull and Nakagami fading channels. Theoretical analysis is presented in this work to show the effects of fading techniques on the BER performance of an OFDM system.

This paper consists of six sections. In Section II, the system model is described. Section III shows various fading channels with their corresponding PDFs. Section IV discusses the performance analysis of OFDM systems with Phase and Frequency Offsets. In Section V, numerical results are presented for Rayleigh, Weibull and Nakagami fading channels. Finally, Section VI presents the conclusion and future work. 


\section{SYSTEM MODEL}

An OFDM system with $\mathrm{N}$ sub-carriers is considered. Let $\mathrm{X}(\mathrm{k})$ be the $\mathrm{k}^{\text {th }}$ OFDM data block to be transmitted with $\mathrm{N}$ subcarriers. These data blocks are used to modulate $\mathrm{N}$ orthogonal subcarriers. Then, using IDFT the input signal is modulated which can be represented as

$$
x(n)=\frac{1}{N} \sum_{k=0}^{N-1} X(k) \exp (i 2 \pi k n / N)
$$

$\mathrm{n}=0,1, \ldots . \mathrm{N}-1$.

Due to the phenomenon of multipath propagation, when the wave hits the obstacle, the signal can be reflected, diffracted or diffused. The channel impulse response of a multipath fading channel is modeled as $|h(n)| e^{-j \theta(n)}$. The receiver side frequency domain signal is represented as

$$
R(k)=X(k) H(k)+N,
$$

where $\mathrm{N}$ is an independent identically distributed (i.i.d) complex Gaussian noise component with zero mean and unit variance. The amplitude of $\mathrm{H}(\mathrm{k})$ is modeled as a Rayleigh, Weibull and Nakagami fading distribution with the corresponding PDFs.

\section{BER analysis in the presence of Offsets} In this section, we discuss BER analysis in the presence of phase and frequency offsets in detail.

\subsection{Carrier Frequency Offset}

The absolute value of the actual CFO, $f_{\varepsilon}$, is either an integer multiple or a fraction of subcarrier spacing, $\Delta f$, or the sum of them. If $f_{\varepsilon}$ is normalized to $\Delta f$, then the resulting normalized $\mathrm{CFO}$ of the channel can be generally expressed as

$$
\varepsilon=\frac{f_{\varepsilon}}{\Delta f}=\delta+\epsilon
$$

where $\delta$ is an integer and $|\in| \leq 0.5$, where

$$
\varepsilon=\frac{\text { frequency offset }}{\text { subcarrier spacing }} \text {. }
$$

The influence of an integer CFO on OFDM system is different from the influence of a fractional CFO. In the event that $\delta \neq 0$ and $\in=0$, symbols transmitted on a certain subcarrier, e.g., subcarrier $k$, will shift to another subcarrier $k_{\delta}$,

$$
k_{\delta}=k+\delta \bmod N-1 \text {. }
$$

As we focus on the ICI effect, we will consider normalized $\mathrm{CFO}, \varepsilon$, since no ICI is caused by an integer CFO. We assume $\varepsilon$ to be a Gaussian process, statistically independent of the input signal with zero mean and variance $\sigma_{\varepsilon}^{2}$. To obtain the preceding expression of probability of error, we consider the PDF of $\varepsilon$ as,

$$
f(\varepsilon)=\frac{1}{\sigma_{\varepsilon} \sqrt{2 \pi}} \exp \left(-\frac{\varepsilon^{2}}{2 \sigma_{\varepsilon}^{2}}\right) \forall \quad \varepsilon>0
$$

and $f(\varepsilon)=0$ for $\varepsilon<0$.

\subsection{Phase Offset}

Phase noise is a random process which results from the fluctuation of the transmitter and receiver Local Oscillators (LO) with time. It also has adverse effect on system performance. Figures 1, 3 and 5 show the resulting probability of error from the phase offset in an OFDM signal. The Signal to Noise Ratio (SNR) of an OFDM signal in the presence of phase noise is obtained.

\subsection{Fading Channels}

The effect of the wireless channel on the transmitted signals is multiplicative, where the multiplicative term is a complex Gaussian random variable. The statistics for fading is very important to design any wireless systems [9]-[14]

\subsubsection{Rayleigh Fading Channel}

If the channel coefficient has zero mean, then such a channel is considered as Rayleigh fading since the absolute value of the received amplitude is a Rayleigh random variable.

The PDF of the received signal amplitude in a Rayleigh fading channel is given as [12, page no. 15]

$$
\mathrm{f}(\mathrm{x})=\frac{\mathrm{x}}{\sigma^{2} \alpha} \exp \left(-\frac{\mathrm{x}^{2}}{2 \sigma^{2} \alpha^{2}}\right) \quad \forall \mathrm{x}>0,
$$

where $\mathrm{x}$ has a Rayleigh Distribution, $\sigma^{2}$ is the variance of received signal amplitude and $\alpha$ is the fading parameter.

\subsubsection{Weibull Fading Channel}

The Weibull distribution is related to a number of other probability distributions; in particular, it interpolates between the exponential distribution $(k=1)$ and the Rayleigh distribution $(k=2)$.

The PDF of the recovered signal in Weibull fading channel is given as [12, page no. 15]

$$
\mathrm{f}(\mathrm{x})=\frac{\mathrm{k}}{\alpha \lambda}\left(\frac{\mathrm{x}}{\alpha \lambda}\right)^{\mathrm{k}-1} \exp \left(-\frac{\mathrm{x}}{\alpha \lambda}\right)^{\mathrm{k}} \forall \mathrm{x}>0 \text {, }
$$

where $k>0$ is the shape parameter, and $\lambda>0$ is the scale parameter of the distribution.

\subsubsection{Nakagami Fading Channel}

The Nakagami-m or Nakagami distribution is a probability distribution related to the Gamma 


$$
f(x)=\frac{2 m^{m}\left(\frac{x}{\alpha}\right)^{2 m-1}}{\Gamma(m) \Omega^{m}} \exp \left(-\frac{m\left(\frac{x}{\alpha}\right)^{2}}{\Omega}\right) \forall x>0 .
$$

distribution. It has two parameters: a shape parameter, $\mu$, and a second parameter controlling spread, $\Omega$. The PDF of the received signal amplitude in a Nakagami fading channel is given as [12, page no. 16]

In the Nakagami fading channel, the fading parameter, $\mathrm{m}$ $=1$, refers to a Rayleigh fading channel. We further note that the system performance analysis with Nakagami fading model is usually more tractable than Rician fading.

\section{PERFORMANCE ANALYSIS OF OFDM SYSTEMS WITH PHASE OFFSET AND FREQUENCY OFFSET}

\subsection{BER Expressions for Phase Offset}

Here, phase offset is assumed to be Gaussian distributed. This assumption is verified by comparing various fading channels with the results of BER calculation [15]-[16]. Phase Offset is represented as . Hex, $\phi \in(0$, $\mathrm{PDF}$ of the phase shift is given as

$$
f(\phi)=\frac{1}{\sigma \sqrt{2 \pi}} \exp \left(-\frac{\phi^{2}}{2 \sigma^{2}}\right) .
$$

(4)

or BPSK Modulation, $\{+1,-1\}$, consider the firs subcarrier transmitted symbol [9]. The constellation is purely real, and the BER is calculated as

$$
\begin{gathered}
B E R=P\left(b_{1}=+1\right) P\left(x<0 \mid b_{1}=+1, \phi\right)+ \\
P\left(b_{1}=-1\right) P\left(x>0 \mid b_{1}=-1, \phi\right) .
\end{gathered}
$$

(5)

The Conditional BER can be written using (5) as

$$
\begin{aligned}
P_{e / \phi}= & \frac{1}{\sigma \sqrt{2 \pi}} \exp \left(-\frac{\phi^{2}(x-1)^{2}}{2 \sigma^{2}}\right) \\
& +\frac{1}{\sigma \sqrt{2 \pi}} \exp \left(-\frac{\phi^{2}(x+1)^{2}}{2 \sigma^{2}}\right) .
\end{aligned}
$$

(6)

\subsubsection{BER Expression for Rayleigh Distribution}

The Probability of Bit Error is given as

$$
P_{e}=\int_{0}^{\infty} \int_{0}^{\pi} p_{e / \phi} f(x) f(\phi) d \phi d x .
$$

(7)
In the Rayleigh case, the conditional probability of BPSK modulation can be written using (6) as

$$
\begin{aligned}
P_{e / \phi}=\frac{1}{\sigma \sqrt{2 \pi}} \int_{-\infty}^{0} \exp \left(-\frac{\phi^{2}(x-1)^{2}}{2 \sigma^{2}}\right) \\
+\frac{1}{\sigma \sqrt{2 \pi}} \int_{0}^{\infty} \exp \left(-\frac{\phi^{2}(x+1)^{2}}{2 \sigma^{2}}\right) .
\end{aligned}
$$

(8)

In (8), using integration by substitution method, we have the results of conditional probability of error obtained as

$$
P_{e / \phi}=\frac{\exp (-\beta)}{\alpha \sigma^{3} \sqrt{2 \pi}}\left[\frac{1}{2 \sqrt{\mu}}+\alpha\left(\frac{\sqrt{\pi}}{2} \operatorname{erf}(v)\right)+\frac{\exp \left(-\mu \alpha^{2}\right)}{\mu}\right] \text {. }
$$

(9)

Substituting (4) and (9) into (7), the unconditional BER is given as

$$
\begin{aligned}
& P_{e}=\int_{0}^{\pi} \frac{\exp (-\beta)}{2 \pi \alpha \sigma^{4}}\left[\exp \left(-\frac{\phi^{2}}{2 \sigma^{2}}\right)\right] \\
& {\left[\frac{1}{2 \sqrt{\mu}}+\alpha \frac{\sqrt{\pi}}{2} \operatorname{erf}(v)+\frac{\exp \left(-\mu \alpha^{2}\right)}{\mu}\right] d \phi . }
\end{aligned}
$$

\subsubsection{BER Expression for Weibull Distribution}

For the Weibull fading case, substituting the PDF of the Weibull fading distribution of (2) into (8), the conditional $\mathrm{BER}$ is given as

$P_{e / \phi}=\frac{2 c^{2} e^{-f}}{\sigma \sqrt{2 \pi}}\left[\int_{-\infty}^{e}(t+e) e^{-d t^{2}} d t+\int_{e}^{\infty}(v-e) e^{-d v^{2}} d v\right]$.

Substituting (4) and (11) into (7), the unconditional BER is given as

$$
\begin{aligned}
P_{e}=\int_{0}^{\pi} \frac{2 c^{2} e^{-f}}{2 \pi \sigma^{2}}\left(\exp \left(-\frac{\phi^{2}}{2 \sigma^{2}}\right)\right) \\
{\left[\int_{-\infty}^{e}(t+e) e^{-d t^{2}} d t+\int_{e}^{\infty}(v-e) e^{-d v^{2}} d v\right] . }
\end{aligned}
$$

\subsubsection{BER Expression for Nakagami Distribution}

In the Nakagami fading case, substituting (8) into (3) using the substitution method, the conditional probability is given as 


$$
P_{e / \phi}=\frac{e^{-p}}{\sigma \sqrt{2 \pi s}}\left[\int_{-\infty}^{s u^{2}} \frac{e^{-r 1}}{\sqrt{r 1}} d r 1+\int_{s u^{2}}^{\infty} \frac{e^{-r 2}}{\sqrt{r 2}} d r 2\right] .
$$

Substituting (4) and (12) into (7), the unconditional BER is given as

$$
\begin{aligned}
& P_{e}=\int_{0}^{\pi} \frac{e^{-p}}{\sigma \sqrt{2 \pi s}}\left(\exp \left(-\frac{\phi^{2}}{2 \sigma^{2}}\right)\right) \\
& {\left[\int_{-\infty}^{s u^{2}} \frac{e^{-r 1}}{\sqrt{r 1}} d r 1+\int_{s u^{2}}^{\infty} \frac{e^{-r 2}}{\sqrt{r 2}} d r 2\right] d \phi . }
\end{aligned}
$$

The derived expressions give the probability of error over various fading channels with phase offset.

\subsection{BER Expressions for Frequency Offset}

Due to spectra overlapping, OFDM systems are sensitive to frequency offset [16]-[17]. The frequency offset destroys the orthogonality among subcarriers, resulting in ICI, and causes system performance degradation. To improve in system performance, the frequency offset must be estimated.The recovered signa $\mathrm{d}_{k}$, on subcarrier $\mathrm{k}$ is given as

$$
d_{k}=I_{0} S_{k}
$$

where $I_{0}$ is the attenuation and phase rotation of the desired signal, $\mathrm{S}_{\mathrm{k}}$ is the $\mathrm{k}^{\text {th }}$ transmitted signal.

From (1), it is clear that the dominant signal on the $\mathrm{k}^{\text {th }}$ subcarrier is $\mathrm{I}_{\mathrm{o}} \mathrm{S}_{\mathrm{k}}$. Hence, the magnitude mean of the dominant signal on the $\mathrm{k}^{\text {th }}$ subcarrier is

$$
m=\left|I_{0} S_{k}\right|=\left|\frac{\sin \pi \varepsilon}{\pi \varepsilon} e^{j \pi \varepsilon} S_{k}\right|=\sin c(\varepsilon),
$$

where $\varepsilon=T_{s} \Delta f \in(-0.5,0.5)$ is the frequency offset normalized to subcarrier spacing. The expectation $d_{k}$ of

$$
\begin{aligned}
& \text { is given as } \mathrm{E}\left(\left|d_{k}\right|\right)=\int_{-\infty}^{\infty} x p_{\left|d_{k}\right|}(x) d x, \\
& \text { i.e, } \\
& \mathrm{E}\left(\left|d_{k}\right|\right)=\sqrt{\frac{2\left(1-\sin c^{2}(\varepsilon)\right)}{\pi}} \exp \left\{-\frac{\sin c^{2}(\varepsilon)}{2\left(1-\sin c^{2}(\varepsilon)\right)}\right\}-\sin c(\varepsilon) .
\end{aligned}
$$

The Second moment of $\left|d_{k}\right|$ is given as

$$
\mathrm{E}\left(\left|d_{k}\right|^{2}\right)=\int_{-\infty}^{\infty} x^{2} p_{\left|d_{k}\right|}(x) d x=1
$$

The Variance of $\left|d_{k}\right|$ can be expressed as

$$
\sigma_{w}^{2}=\mathrm{E}\left(\left|d_{k}\right|^{2}\right)-\mathrm{E}^{2}\left(\left|d_{k}\right|\right),
$$

i.e,

$$
\sigma_{w}^{2}=1-\left[\sqrt{\frac{2\left(1-\sin c^{2}(\varepsilon)\right)}{\pi}} \exp \left\{-\frac{\sin c^{2}(\varepsilon)}{2\left(1-\sin c^{2}(\varepsilon)\right)}\right\}-\sin c(\varepsilon)\right]^{2} .
$$

Based on the above analysis, the PDF of the output, can be approximated as a Gaussian mixture as

$$
p_{\left|d_{k}\right|}(x)=\left[\frac{1}{\sigma_{w} \sqrt{2 \pi}} e^{-(x-m)^{2} / 2 \sigma_{w}{ }^{2}}+\frac{1}{\sigma_{w} \sqrt{2 \pi}} e^{-(x+m)^{2} / 2 \sigma_{w}{ }^{2}}\right] u(x),
$$

where $\mathrm{u}(\mathrm{x})$ is the unit step function.

$$
P_{b}(\varepsilon)=\int_{o}^{\infty}\left[\frac{1}{\sigma_{w} \sqrt{2 \pi}} e^{-(x-m)^{2} / 2 \sigma_{w}{ }^{2}}+\frac{1}{\sigma_{w} \sqrt{2 \pi}} e^{-(x+m)^{2} / 2 \sigma_{w}{ }^{2}}\right] f(x) d x,
$$

where $f(x)$ represents the PDF of various fading distributions, and (16) gives the BER conditioned on the normalized frequency offset, $\in$. The unconditional BER is then given as

$$
P_{e}=\int_{0}^{\infty} \int_{\in=-0.5}^{0.5} p_{\left|d_{k}\right|}(x) f(x) f(\varepsilon) d \varepsilon d x
$$

\section{NUMERICAL RESULTS}

As expected, all results show that BER increases with increase in Phase and Frequency Offsets. Figures 1, 3 and 5 show that BER increases with increase in phase offset (increasing value of $\sigma$ ) over Rayleigh, Weibull and Nakagami fading. Figures 2, 4 and 6 show that the performance of the BER decreases as SNR increases. The effect of Normalized CFO gives the BER performance of BPSK modulation scheme as illustrated in Figures 7, 8 and 9. Figures 7, 8 and 9 show that BER increases with increase in Frequency offset

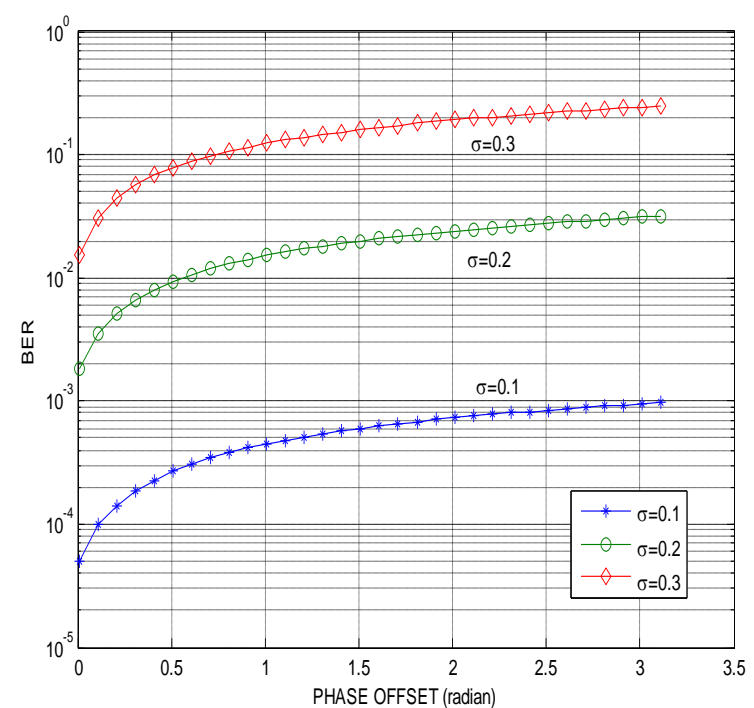


Figure 1: BER vs. phase offset with Gaussian Distribution over a Rayleigh fading channel

Figure 1 shows the performance of the BER as a function of Phase Offset over a Rayleigh fading channel. Here, the BER increases from $10^{-4.6}$ to $10^{-3}$ for the variance of $\sigma=0.1$ as the Phase offset varies between $(0, \pi)$ radians. Also, as the variance increases from $\sigma=0.1,0.2,0.3$, the BER increases from $10^{-4.6}$ to $10^{-0.85}$ This results in degradation of system performance with increase in phase offset.

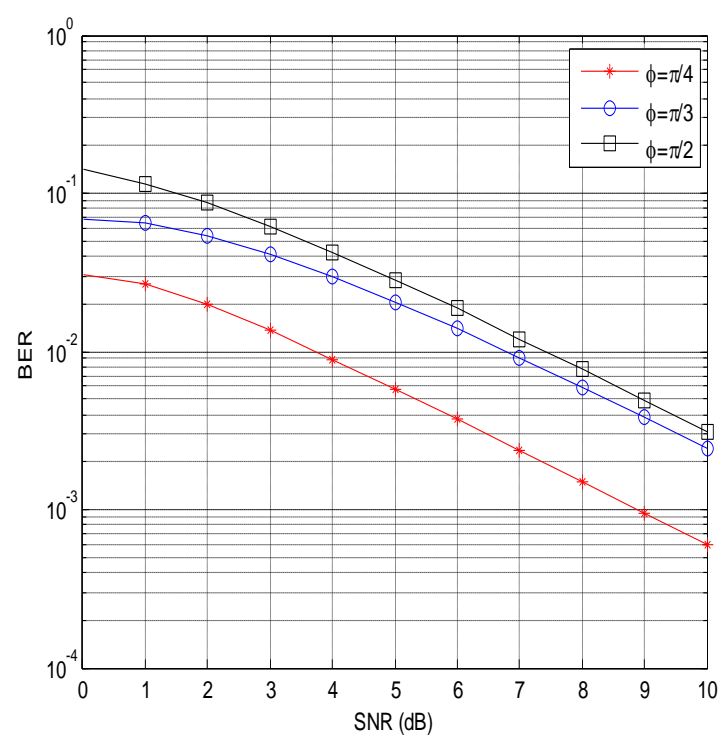

Figure 2: BER vs. SNR with Gaussian distribution over a Rayleigh fading channel

Figure 2 shows the performance of BER versus SNR over a Rayleigh fading channel. Here, the BER decreases from $10^{-1.8}$ to $10^{-3.5}$ for a phase of $\phi=\pi / 4$ as SNR increases from $0 \mathrm{~dB}$ to $10 \mathrm{~dB}$. The BER value increases from $10^{-1.8}$ to $10^{-0.95}$ with increase in phase offset, $\phi=\frac{\pi}{4}, \frac{\pi}{3}, \frac{\pi}{2}$.

For the SNR value of $5 \mathrm{~dB}$, as the phase decreases from $\phi=\frac{\pi}{2}$ to $\frac{\pi}{4}$, BER also decreases from $10^{-1.8}$ to $10^{-2.5}$.

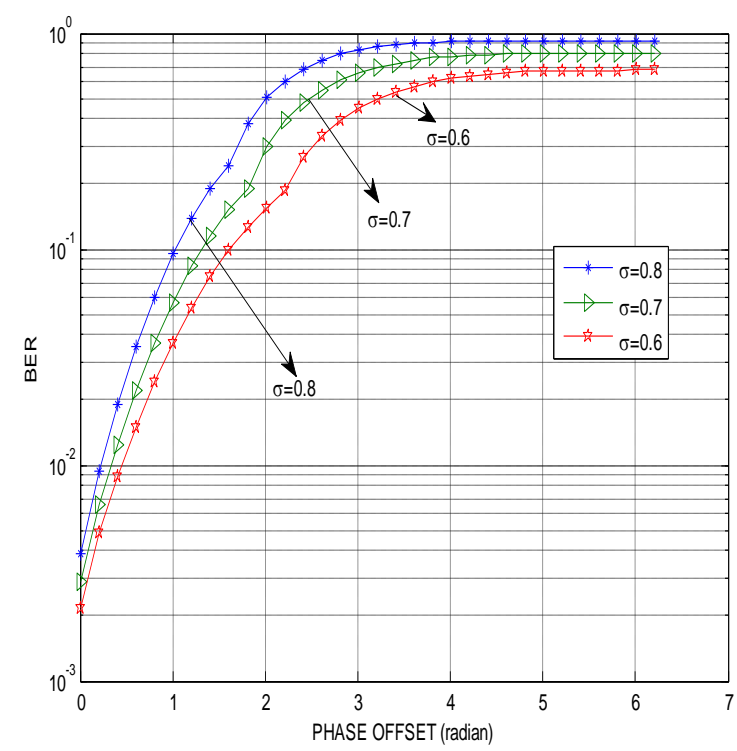

Figure 3: BER vs. Phase Offset with Gaussian distribution over a Weibull fading channel

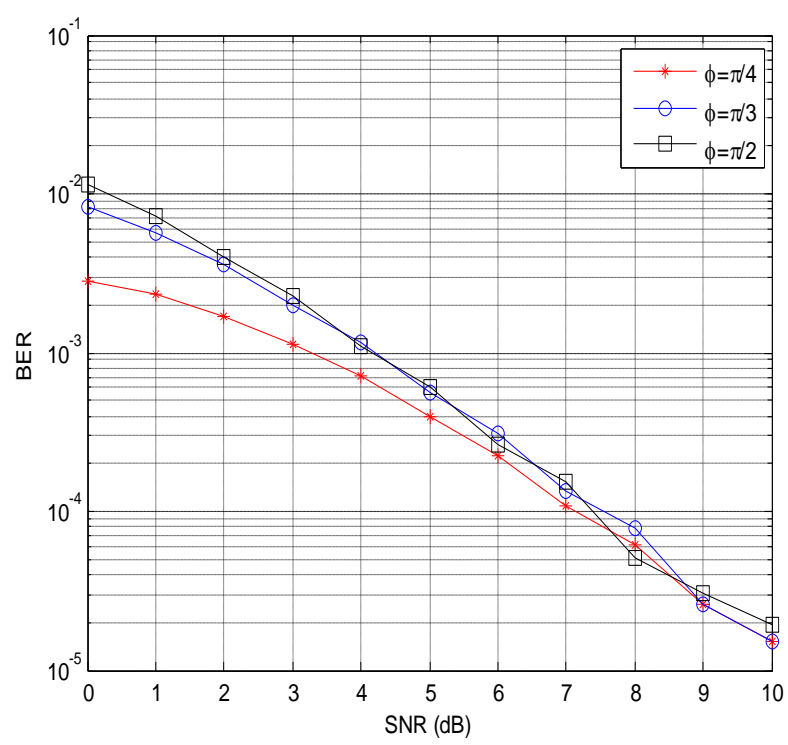

Figure 4: BER vs. SNR with Gaussian distribution over a Weibull fading channel

Figure 3 shows the performance of BER versus Phase Offset over a Weibull fading channel. When the variance is assumed to be $\sigma=0.6$, BER increases from $10^{-2.9}$ to $10^{-0.4}$ with an increase in phase offset by $2 \pi$ (radian). For different values of variance, $\sigma=0.6,0.7,0.8$ an increase in the BER curve from $10^{-2.9}$ to $10^{-2.7}$ is observed. This degrades the system performance considerably when an increase in phase offset is experienced. 
Figure 4 shows the performance of BER versus SNR over a Weibull fading channel. It is inferred that BER decreases from $10^{-2}$ to $10^{-4.9}$ with an increase in SNR from $0 \mathrm{~dB}$ to $10 \mathrm{~dB}$ for the phase value of $\phi=\pi / 2$. The BER value increases from $10^{-2.8}$ to $10^{-2}$ as the Phase offset takes values, $\phi=\frac{\pi}{4}, \frac{\pi}{3}, \frac{\pi}{2}$. For the SNR value of $2 \mathrm{~dB}$, a decrease in phase from $\phi=\frac{\pi}{2}$ to $\frac{\pi}{4}$ results in a decrease in BER from $10^{-2.7}$ to $10^{-2.85}$ is observed.

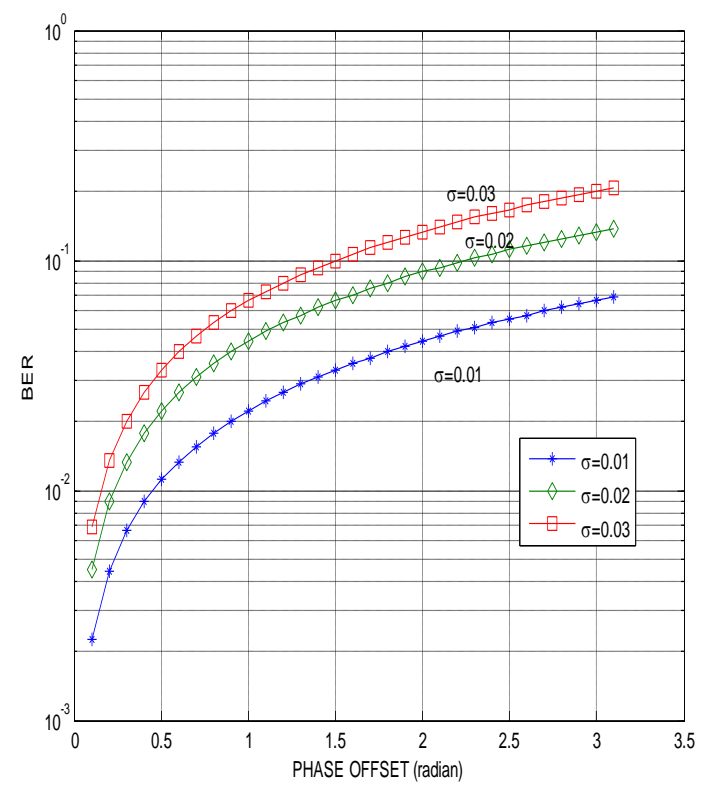

Figure 5: BER vs. Phase Offset with Gaussian distribution over a Nakagami fading channel

Figure 5 shows the performance of BER versus phase offset over a Nakagami fading channel. Here, the BER increases from $10^{-2.9}$ to $10^{-1.4}$ for the variance $\sigma=0.01$ as the Phase offset increases. Also, variance $\sigma=0.01,0.02,0.03$ increases as BER increases from $10^{-2.9}$ to $10^{-2.4}$. This results in system degradation with increase in phase offset.

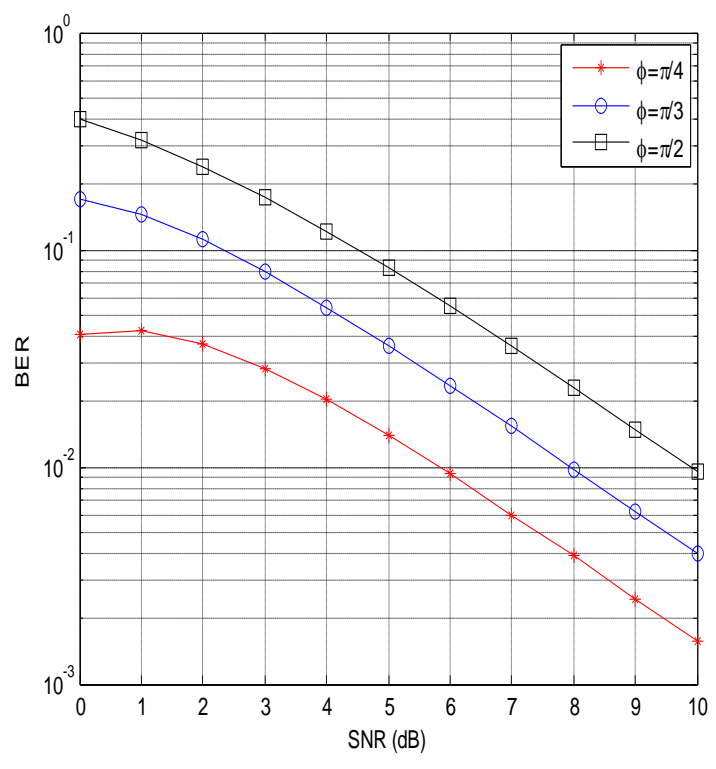

Figure 6: BER vs. SNR with Gaussian distribution over a Nakagami fading channel

Figure 6 shows the performance of BER vs. SNR over a Nakagami fading channel. Here, the BER decreases from $10^{-0.7}$ to $10^{-2.1}$ as SNR increases $10 \mathrm{~dB}$ for the phase value of $\phi=\pi / 2$. The BER value increases from $10^{-1.7}$ to 10 ${ }^{0.7}$ with increase in phase offset $\phi=\frac{\pi}{4}, \frac{\pi}{3}, \frac{\pi}{2}$. For the SNR value $5 \mathrm{~dB}$, as the phase decreases from $\phi=\frac{\pi}{2}$ to $\frac{\pi}{4}$, the BER also decreases from $10^{-1.3}$ to $10^{-}$ 1.95 .

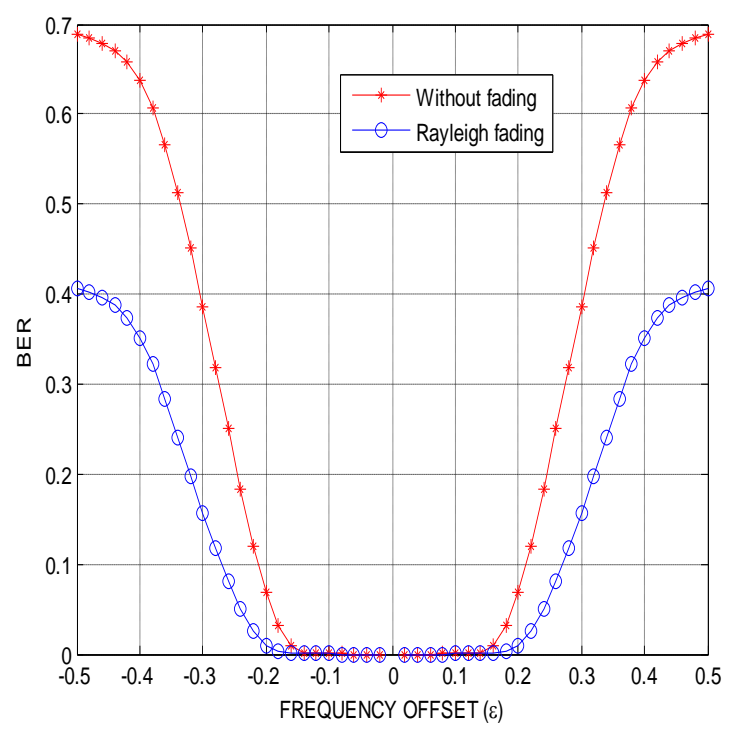

Figure 7: BER vs. Frequency Offset with Gaussian distribution over a Rayleigh fading channel

Figure 7 shows the performance of BER vs. Frequency offset over a Rayleigh fading channel. Here, for the no fading case, BER increases from 0.0013 to 0.4 with increase in Frequency offset in $(-0.5,0.5)$. For the 
Rayleigh fading case, BER increases from 0.0011 to 0.7 with increase in the Frequency offset in $(-0.5,0.5)$.

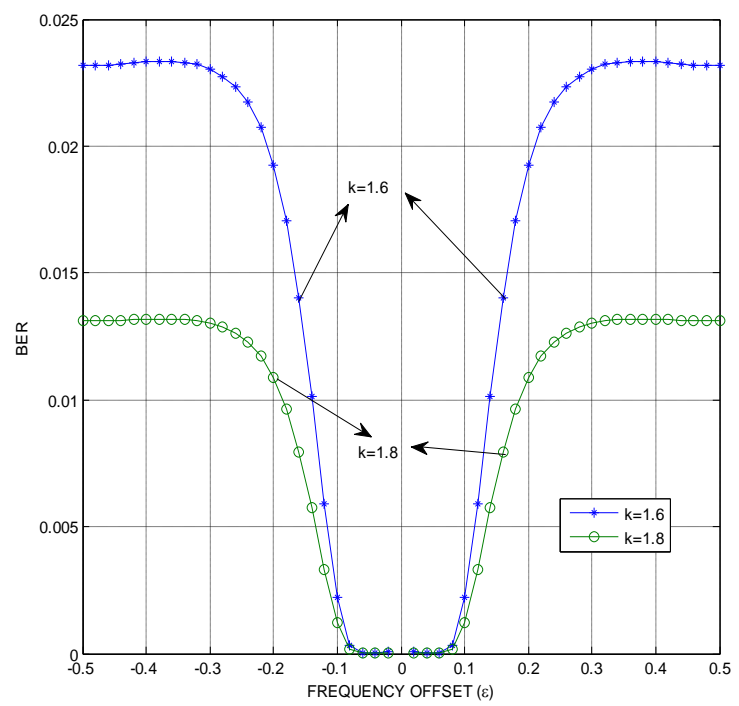

Figure 8: BER vs. Frequency Offset with Gaussian distribution over a Weibull fading channel

Figure 8 shows the performance of BER vs. Frequency offset over Weibull fading channel. Here, the BER increases from 0.013 to 0.023 with increase in Frequency offset $(-0.5,0.5)$

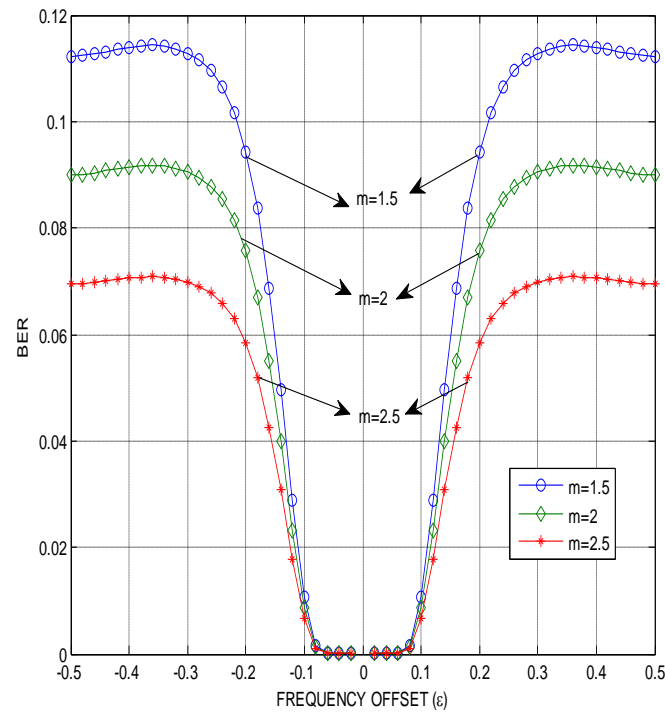

Figure 9: BER vs. Frequency Offset with Gaussian distribution over a Nakagami fading channel

Figure 9 shows the performance of the BER versus Frequency offset over a Nakagami fading channel. Here, the BER increases from 0.07 to 0.12 with increase in Frequency offset in $(-0.5,0.5)$.

\section{CONCLUSIONS}

In this paper, we have analytically evaluated the performance of an OFDM system using BPSK modulation with Phase and Frequency Offsets over various fading channels. It can be observed that system performance severely degrades with increase in these offsets. The analytical expressions obtained closely match the Numerical results. Graphical results show improvement in OFDM BPSK system with decrease in Phase and Frequency Offsets in Rayleigh, Weibull and Nakagami fading channels. The reported BER can be further reduced by using channel estimation or suitable diversity schemes, and is the focal point of future research work.

\section{REFERENCES}

[1] IEEE, "Wireless LAN Medium Access Control (MAC) and Physical Layer (PHY) Specifications: High-speed Physical Layer in the $5 \mathrm{GHz}$ Band," IEEE Standard 802.11a, 1999.

[2] IEEE, "Wireless LAN Medium Access Control (MAC) and Physical Layer (PHY) Specifications Amendment 4: Further Higher Data Rate Extension in the $2.4 \mathrm{GHz}$ Band," IEEE Standard 802.11g, 2003.

[3] F. Yang, K. H. Li, and K. C. Teh, "Performance Analysis of the Blind Minimum Output Variance Estimator for Carrier Frequency Offset in OFDM Systems," EURASIP Journal on Advances in Signal Processing, pp. 1-8, Sep. 2006.

[4] L. Rugini and P. Banelli, "BER of OFDM systems impaired by carrier frequency offset in multipath fading Channels," IEEE Transactions on Wireless Communications, vol. 4, no. 11 , pp. 2279-2288, Sep. 2005.

[5] T. Pollet, M. Van Bladel, and M. Moeneclaey, "BER sensitivity of OFDM systems to carrier frequency offset and Wiener phase noise," IEEE Transactions on Communications, vol. 43, pp. 191-193, Feb./Mar./Apr. 1995.

[6] H. Moose, "A technique for orthogonal frequency division multiplexing frequency offset correction," IEEE Transactions on Communications, vol. 42, no 10, pp. 2908-2914, Oct. 1994.

[7] P. Dharmawansa, N. Rajatheva, and H. Minn, "An exact error probability analysis of OFDM systems with frequency offset," IEEE Transactions on Communications, vol. 57, no. 1, pp. 26-31, Jan. 2009.

[8] S. Mallick and S. P. Majumder, "Performance analysis of an OFDM system in the presence of carrier frequency offset, phase noise and timing jitter over rayleigh fading channels,"

[9] International Conference on Electronics and Communication Engineering, Dhaka, Bangladesh, vol. 5, no. 12, pp. 205-210, Dec. 2008.

[10] T. S. Rappaport, Wireless Communications, Principles and Practice, 2nd edition. Prentice Hall Inc. NJ, 2002

[11] A. Papoulis, Probability, Random Variables and Stochastic Processes, 3rd edition, McGraw-Hill Inc. NY, 1991.

[12] I. S. Gradshteyn and I. M. Ryzhik, Table of Integrals, Series, and Products, 7th edition San Diego, CA: Academic, 2000. 
[13] M. K. Simon and M. S. Alouini, Digital Communication Over Fading Channels, 2nd edition, Wiley, NJ, 2005.

[14] Tolga M. Duman, and Ali Ghrayeb, Coding for MIMO Communication systems, 3rd edition John Wiley \& Sons Ltd., 2007.

[15] J.G. Proakis, Digital Communications, 4tth edition, McGraw-Hill Inc. NY, 2001.

[16] S. Wu and Y. Bar-Ness, "OFDM Systems in the Presence of Phase Noise: Consequences and Solutions," IEEE Transactions on Communication, vol. 52, no. 11, pp. 1988-1996, Nov. 2004.
[17] A. Armada, "Understanding the Effects of Phase Noise in Orthogonal Frequency Division Multiplexing (OFDM)," IEEE Transactions on Broadcasting, vol. 47, no. 2, pp. 153-159, June 2001.

[18] K. Sathananthan and C. Tellambura, "Probability of error calculation of OFDM systems with frequency offset," IEEE Transactions on Communications, vol. 49, no. 11, pp. 1884-1888, Nov. 2001.

[19] N. C. Beaulieu and P. Tan, "On the effects of receiver windowing on OFDM performance in the presence of carrier frequency offset," IEEE Transactions on Wireless Communications, vol. 6, no. 1, pp. 202-209, Jan. 2007. 